\author{
REVISTA SABERES EDUCATIVOS \\ DICIEMBRE 2018, $\mathrm{N}^{\circ}$ 2, 13-37
}

\title{
LA COMPETENCIA COMUNICATIVA Y LOS ESTILOS DE APRENDIZAJE EN LA FORMACIÓN DE PROFESIONALES DEL ÁREA EDUCACIÓN Y CIENCIAS ECONÓMICAS ${ }^{1}$
}

\author{
María Elena Arriagada Arriagada ${ }^{2}$ \\ Natalia Henríquez Cabezas ${ }^{3}$
}

RESUMEN/ ABSTRACT

El presente artículo analiza la relación entre los estilos de aprendizaje (reflexivo, activo, teórico y pragmático) y la secuencia explicativa en la acción expositiva oral universitaria. Así mediante una matriz analítica se evalúan las presentaciones orales de 16 estudiantes de la carrera de Pedagogía en Educación Básica y 16 de la carrera de Ingeniería Comercial y se compara con los indicadores obtenidos de la aplicación del Cuestionario de Estilos de Aprendizaje (Alonso, Gallego y Honey, 1994). Los resultados muestran que los/as estudiantes de la carrera de educación consiguieron un mejor desempeño discursivo en cuanto a estructura y recursos en la tarea expositiva, lo que refrenda la hipótesis de la temprana transferencia de los modelos y esquemas cognitivo-discursivo del área de educación a los/as estudiantes noveles. Sin embargo, el análisis correlacional entre estilos de aprendizaje y el desempeño del discurso expositivo no dan resultados significativos.

Palabras claves: competencia comunicativa oral, formación universitaria, estrategias explicativas, estilos de aprendizaje.

This article analyzes the connection between different learning styles (reflexive, active, theoretical and pragmatic) and the explanatory sequence in oral presentations at university.

\footnotetext{
${ }^{1}$ Este trabajo se enmarca en el desarrollo del Proyecto FGI 12-16: "Aprender a aprender en la Universidad, un desafío para los/as estudiantes de primer año de la carrera Licenciatura en Educación y Pedagogía Básica" con financiamiento de la Dirección de Investigación de la Universidad Metropolitana de Ciencias de la Educación (DIUMCE) años 2016-2017.

${ }^{2}$ Académica de la Facultad de Filosofía y Educación, Departamento de Educación Básica, Universidad Metropolitana de Ciencias de la Educación (UMCE); Doctora en Lingüística.; m_elena.arriagada@umce.cl

3 Académica de la Facultad de Ciencias, Departamento de Matemática, Universidad Metropolitana de Ciencias de la Educación (UMCE); Doctora en Estadística; natalia.henriquez_c@umce.cl
} 
Thus, using an analytic matrix, 16 oral presentations of students of the program of Primary School Teaching and 16 of the program of Economic Sciences are analyzed and compared with the indicators obtained from the Questionnaire of Learning Styles (Alonso, Gallego and Honey, 1994). The results show that the students of the teaching program had a better discursive performance regarding structure and resources in the expository task, which confirms the hypothesis of early transfer of models and cognitive-discursive schemes to the new students of the area of education. However, the correlation analysis between learning styles and the discursive elements does not give significant results.

Key words: oral communicative competence; university education; explanatory strategies; learning styles.

\section{INTRODUCCIÓN}

En los últimos años, los requerimientos de la formación universitaria han develado una ingente necesidad de modelar la eficacia comunicativa de la oralidad, en el marco de la formación por competencias. La incorporación de métodos y modelos tomados desde varias disciplinas de la lingüística ha hecho posible realizar precisiones conceptuales específicas sobre la eficacia de la comunicación en este contexto y, a su vez, explicitar la incorporación de competencias cognitivas e interpersonales.

Una de las dificultades en el contexto disciplinar académico es que se suele dirigir la enseñanza-aprendizaje al resultado y no al proceso que esta supone. Para que esto no ocurra, el docente debe, primeramente, diagnosticar y atender a los procesos cognitivos y conceptuales, las formas de habla, los estilos de aprendizajes, entre otros aspectos, a tener en cuenta. Posteriormente, debe propiciar oportunidades de aprendizaje, tanto individual como grupal, lo que implica implementar estrategias adecuadas y específicas al contexto. Dichas estrategias permiten hacerse cargo del "déficit académico" y facilitar el tránsito formativo a las exigencias académicas instaladas en los primeros años de las distintas carreras, atendiendo a los perfiles de egreso.

En este estudio se entienden los estilos de aprendizaje como: “(...) variables personales que, a mitad de camino entre la inteligencia y la personalidad, explican las 
diferentes formas de abordar, planificar y responder ante las demandas del aprendizaje" (Camarero, Buey y Herrero 2000, p.615). A partir de esta definición, la teoría de los estilos de aprendizaje ofrece indicadores de los canales de preferencias (activo, reflexivo, teórico y pragmático) de los sujetos para seleccionar, procesar y organizar la información recibida. Estos indicadores son de utilidad tanto para los/las docentes, en las planificaciones e implementación de las mismas, como para los/las estudiantes, en la reflexión y autorregulación del propio proceso de aprendizaje, ya que su conocimiento estimula el "aprender a aprender", esto es, que el/la aprendiz sea capaz de autodirigir su aprendizaje y transferirlo a otros ámbitos de su vida. Todo lo anterior, en el marco de una formación integral de especialistas competentes en las distintas disciplinas (Vilà i Santasusana, 2005; Manzano e Hidalgo, 2009; González, 2011).

La falta de desarrollo de la competencia comunicativa en los/las estudiantes puede convertirse en una limitante para sus aspiraciones académicas. Por ello, es importante la participación activa de el/la aprendiz en la reflexión sobre sí mismo como estudiante (Martín y Moreno, 2007), ya que el desarrollo de la expresión oral requiere de una preparación sistemática, holística y permanente.

Según sostiene Helena Calsamiglia (1994), la expresión oral exige de los/las estudiantes una serie de habilidades específicas para la elaboración de los discursos orales, entre ellas, ser coherente en la distribución de los contenidos, dominar el uso de la cohesión para plantear las ideas y, además, ser adecuado a los diversos contextos comunicativos. Para ello el/la hablante debe configurar esquemas cognitivos-discursivos mediante operaciones proposicionales que organizan una secuencia prototípica, como lo es, por ejemplo la explicación (Zamudio y Atorresi, 2000; Casco, 2000).

Es en este marco que nos planteamos indagar sobre los estilos de aprendizaje, su relación con el uso de estrategias discursivas explicativas y su influencia en el desarrollo de la competencia comunicativa oral de estudiantes universitarios de primer año de las carreras de Pedagogía en Educación Básica y de Ingeniería Comercial provenientes de universidades chilenas en una tarea expositiva de aula con grabación de video como herramienta de evaluación. 
Para tales propósitos este estudio aplica el Cuestionario de Estilos de Aprendizaje que consta de 80 ítems de respuesta dicotómica, de las cuales 20 corresponden a cada estilo de aprendizaje y se distribuyen de forma aleatoria. La puntuación que cada sujeto obtiene en cada grupo de 20 ítems indica la calificación que alcanza en cada estilo de aprendizaje: activo, pragmático, reflexivo y teórico. Además, se pudo observar, registrar en video y analizar el empleo del esquema discursivo explicativo sobre un tema establecido (Zamudio y Torresi, 2000) en 32 exposiciones orales (16 respectivamente para cada carrera), cuya duración es de 8 a 10 minutos cada una.

De esta manera, para establecer la existencia de vinculación entre los estilos de aprendizaje y las estrategias explicativas discursivas utilizadas por estos/as estudiantes se aplicó una metodología cuantitativa a través de un análisis correlacional, donde se utiliza el test de Chi-cuadrado de Pearson. No obstante, dadas las limitaciones en el acceso a la información y la cantidad de esta, el presente estudio se enmarca dentro de una investigación pseudoexperimental, que tiene el propósito de establecer si existen diferencias entre los estilos de aprendizaje y el desempeño discursivo explicativo, dadas las características disciplinares de las carreras.

A continuación, en primer lugar, se presentan los antecedentes teóricos de la investigación; en segundo lugar, se expone el diseño metodológico que proporciona respuestas al proceso investigativo, particularmente, al objetivo e hipótesis del mismo; y, finalmente, se señalan las conclusiones a las que se arribó.

\section{ANTECEDENTES TEÓRICOS DE LA INVESTIGACIÓN}

En las universidades chilenas se ha instalado el debate acerca de cómo realizar de forma efectiva un Aprendizaje Basado en Competencias (ABC). Tomado desde el marco europeo de la buena enseñanza, el ABC supone una importante innovación en la formación de los/as jóvenes, que pone el acento en habilidades necesarias para el desempeño y adaptación a las complejas circunstancias de nuestro medio social. Tal como lo describe un grupo de investigación sobre $\mathrm{ABC}$ de la Universidad de Deusto (España), este consiste en: 
(...) desarrollar las competencias genéricas o transversales (instrumentales, interpersonales y sistémicas) necesarias y las competencias específicas (propias de cada profesión) con el propósito de capacitar a la persona sobre los conocimientos científicos y técnicos, su capacidad de aplicarlos en contextos diversos y complejos, integrándolos con sus propias actitudes y valores en un modo propio de actuar personal y profesionalmente" (Villa y Poblete 2007, p.30).

En consecuencia, lo que el $\mathrm{ABC}$ considera es que, a fin de conseguir una exitosa participación y vinculación en nuestro mundo contemporáneo, ya no bastan los conocimientos y saberes adquiridos a partir de la formación inicial universitaria; por el contrario, frente a diferentes situaciones académicas y/o profesionales, resulta más importante la capacidad de cada individuo de vincular sus habilidades intersubjetivas y sus recursos de pensamiento (López, 2013). Cabe señalar que en relación al ABC, uno de los aspectos que genera mayor controversia en las universidades chilenas es la complejidad de su implementación dada la divergencia existente entre la heterogeneidad de los/as estudiantes de primer año y los estándares que declaran los perfiles de egreso de las carreras (Latorre, Aravena, Milos y García, 2010).

Dentro de las competencias genéricas del modelo $\mathrm{ABC}$ se sitúa la noción de competencia comunicativa, descrita por Hymes (1972) como una habilidad transversal, modelada por la experiencia y vinculación con el entorno y que, por tanto, constituye una herramienta base para el desempeño eficaz de los roles profesionales:

La competencia comunicativa es el término más general para la capacidad comunicativa de una persona, capacidad que abarca tanto el conocimiento de la lengua como la habilidad para utilizarla. La adquisición de tal competencia está mediada por la experiencia social, las necesidades y motivaciones, y la acción, que es a la vez una fuente renovada de motivaciones, necesidades y experiencias. (Hymes 1964, p.31).

Lo cierto es que para el desarrollo de dicha competencia, los/as alumnos/as llevan a cabo de manera continua, desde sus primeros años en el sistema educacional chileno, prácticas de comunicación oral, de lectura y de escritura. Sin embargo, el ingreso a la vida 
universitaria implica un proceso de aprendizaje que contempla contenidos disciplinarios complejos, que requieren por parte de los/as docentes estrategias de enseñanza apropiadas, así como también, de materiales didácticos y sistemas de evaluación que contemplen el desarrollo de habilidades específicas. A juicio de los/as expertos, las materias universitarias plantean "nuevos desafíos y exigen continuar aprendiendo a leer y escribir" (Carlino 2005, p.4), no solo a nivel de educación superior, sino también a nivel profesional, pues son procesos perfectibles, por lo que es necesario que la institución formativa apoye y se hago cargo del desarrollo de estrategias de comprensión y producción de textos disciplinares.

Es en el aula universitaria donde deben enseñarse los usos lingüísticos y discursivos para la producción de textos académicos y disciplinares, de modo que los/las estudiantes puedan acercar sus intentos discursivos a los de el/la especialista. Desde la perspectiva docente y desde el currículo, la exposición oral como estrategia metodológica en el desarrollo de la competencia comunicativa es recurrente, pues el hablar sirve para que el estudiantado se apropie y comparta el conocimiento disciplinar a través de las prácticas discursivas orales de la academia. Según sostiene la investigadora Paula Carlino:

(...) alfabetizar académicamente equivale a ayudar a participar en prácticas discursivas contextualizadas, lo cual es distinto de hacer ejercitar habilidades desgajadas que fragmentan y desvirtúan esas prácticas. Porque depende de cada disciplina y porque implica una formación prolongada, no puede lograrse desde una única asignatura ni en un sólo ciclo educativo. Así, las "alfabetizaciones académicas" incumben a todos los docentes a lo ancho y largo de la universidad. (Carlino 2013, p. 370).

Además, Tomlin, Forrest, Pu y Kim (1997) sostienen que en la producción del discurso, los/as hablantes deben enfrentarse, por un lado, al problema de la integración del conocimiento, es decir, la selección y organización de experiencias relevantes y pertinentes que sirvan de apoyo a la comprensión del interlocutor y, por otro, al problema de la gestión de la información, esto es, el manejo de expectativas psicocomunicativas que supeditan la selección de materiales informativos bajo reglas basadas no solo en las estructuras lingüísticas, sino también en aquellas con base en la cognición social, que es el área de estudio del procesamiento mental de la información sobre el medio social, de manera que la 
demanda de ciertas secuencias recurrentes permiten reducir el esfuerzo de procesamiento de información por parte de los oyentes cuyos esquemas ya están almacenados (Van Dijk y Kinsch, 1983; Gallardo-Paúls, 2007).

En el presente trabajo abordamos, específicamente, la exposición oral como un esquema cognitivo-discursivo, que exige como tarea la elaboración, selección y organización de materiales pertinentes para dar cuenta de una representación conceptual interiorizada por parte del hablante. Lo anterior nos permite no solo producir tipos textuales concretos, sino también generar expectativas sobre el modo en que debería estar organizada la información cuando la procesamos. Como fenómeno discursivo, "la explicación" supone un agente poseedor de un saber y una audiencia o interlocutor que está en disposición de interpretar a partir de conocimiento previo que posee, pero que necesita aclaración para lograr comprender. De ahí que consideramos la secuencia explicativa (Adam,1992; Zamudio y Atorresi, 2000), ya que, desde el punto de vista de la organización textual, requiere de orden y claridad para establecer una lógica del conjunto de enunciados.

\section{MACROPROPOSICIÓN EXPLICATIVA}

Esquematización inicial: se presenta un objeto complejo inicial, cuyos caracteres están dados como hechos establecidos a partir de expresiones del tipo "como todos sabemos", "se ha observado", "se ha percibido".

Problema: el objeto complejo inicial se transforma en objeto problematizado, a través de los operadores "por qué" o "cómo".

Explicación: se integran nuevos antecedentes lógicos que transforman el objeto complejo inicial en objeto explicado a través del operador "porque".

Conclusión: se demuestra o ratifica la validez de la explicación, a través del conector "en realidad". 
Tabla 1. Matriz Analítica de esquema discursivo

\begin{tabular}{|c|c|c|c|c|}
\hline & $\begin{array}{c}\text { Esquematización (1) } \\
\text { Inicial }\end{array}$ & Problema (2) & Explicación (3) & Conclusión (4) \\
\hline 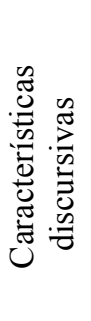 & $\begin{array}{l}\text { Se presenta un objeto } \\
\text { complejo inicial, cuyos } \\
\text { caracteres están dados } \\
\text { como hechos } \\
\text { establecidos a partir de } \\
\text { expresiones }\end{array}$ & $\begin{array}{c}\text { El objeto } \\
\text { complejo } \\
\text { inicial se } \\
\text { transforma en } \\
\text { objeto } \\
\text { problematizado }\end{array}$ & $\begin{array}{l}\text { Se integran } \\
\text { nuevos } \\
\text { antecedentes } \\
\text { lógicos que } \\
\text { transforma el } \\
\text { objeto complejo } \\
\text { inicial, en objeto } \\
\text { explicado }\end{array}$ & $\begin{array}{l}\text { Se demuestra o } \\
\text { ratifica la } \\
\text { validez de la } \\
\text { explicación. }\end{array}$ \\
\hline 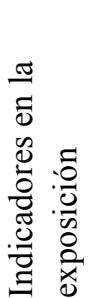 & $\begin{array}{l}\text { Presentación del tema, } \\
\text { contextualización. } \\
\text { Marcadores discursivos } \\
\text { del tipo "como todos } \\
\text { sabemos", "se } \\
\text { observado", "se } \\
\text { percibido". }\end{array}$ & $\begin{array}{l}\text {, a través de los } \\
\text { operadores por } \\
\text { qué o cómo }\end{array}$ & $\begin{array}{l}\text { A través del } \\
\text { operador } \\
\text { porque. }\end{array}$ & $\begin{array}{l}\text { A través del } \\
\text { conector, } \\
\text { realidad }\end{array}$ \\
\hline
\end{tabular}

\section{MARCO METODOLÓGICO}

\subsection{OBJETIVO}

Conocer la relación entre la secuencia explicativa, estilos de aprendizaje y competencia comunicativa en estudiantes noveles del área educación y ciencias económicas.

\section{$2.2 \quad$ PARTICIPANTES}

Este estudio se realizó con estudiantes de primer año de la cohorte 2016, cuyas edades en su mayoría fluctuaban entre los 18 y 20 años, pertenecientes a las carreras de Ingeniería Comercial de la Universidad Bernardo O’Higgins y de Pedagogía en Educación Básica de la Universidad Metropolitana de Ciencias de la Educación. Las muestras recogidas fueron no aleatorias, con un tamaño de 16 integrantes por cada carrera, de un total de 50 respectivamente. 


\section{$2.3 \quad$ INSTRUMENTOS}

En primera instancia se aplicó el Cuestionario de Estilos de Aprendizaje (CHAEA) de manera presencial y en un periodo de 15 a 20 minutos, con el objetivo de constatar diferencias en los estilos de aprendizaje de ambos grupos de estudiantes, lo cual permite establecer esquemas de comportamiento de estos que, a su vez, inciden directamente con los procesos de enseñanza-aprendizaje (Gutiérrez, García, Vivas, Santizo, Alonso y Arranz, 2011). La asignación del estilo de aprendizaje se realizó finalmente con el esquema Baremo que proponen Alonso et al. (1994) dado que, como se muestra en la Tabla 2, las puntuaciones entre los diferentes estilos no son equivalentes.

Tabla 2. Baremo General de Preferencia de Estilos de Aprendizaje

\begin{tabular}{lccccc}
\hline & $\begin{array}{c}10 \% \\
\text { Preferencia } \\
\text { Muy baja }\end{array}$ & $\begin{array}{c}20 \% \\
\text { Preferencia } \\
\text { Baja }\end{array}$ & $\begin{array}{c}40 \% \\
\text { Preferencia } \\
\text { Moderada }\end{array}$ & $\begin{array}{c}\text { Preferencia } \\
\text { Alta }\end{array}$ & $\begin{array}{c}\text { Preferencia } \\
\text { Muy Alta }\end{array}$ \\
\hline Activo & $0-6$ & $7-8$ & $9-12$ & $13-14$ & $15-20$ \\
Reflexivo & $0-10$ & $11-13$ & $14-17$ & $18-19$ & 20 \\
Teórico & $0-6$ & $7-9$ & $10-13$ & $14-15$ & $16-20$ \\
Pragmático & $0-8$ & $9-10$ & $11-13$ & $14-15$ & $16-20$ \\
\hline
\end{tabular}

Fuente: Alonso et al., 1994: 114

Por otra parte, a ambos grupos de estudiantes se les encomendó la tarea de presentar diversos temas individuales de acuerdo a su especialización, es decir, temas relacionados en el ámbito de las ciencias económicas para el grupo de estudiantes de Ingeniería Comercial y para el grupo de Educación en Pedagogía Básica temas relacionados con la enseñanza y aprendizaje.

Se asignó un tiempo adecuado para la preparación de dichos temas y la presentación de estos fue evaluada mediante la matriz analítica (Tabla 1), la cual fue dada a conocer con anterioridad a los/as estudiantes. Las presentaciones respectivas fueron grabadas para su posterior evaluación. En este punto cabe destacar el uso de las TIC (Tecnologías de la 
Información y la Comunicación) no solo como recurso utilizado por los/las estudiantes para sus exposiciones, sino principalmente por las utilidades que reporta la grabación de video como estrategia de evaluación, ya que permite procesos de autoobservación y de reflexión sobre la propia actuación de las habilidades comunicativas. De este modo, la integración de esta herramienta en el proceso de enseñanza aprendizaje favorece que los/las estudiantes tomen conciencia de las mejoras que deben realizar para el desarrollo de sus competencias comunicativas, considerando además las valoraciones de sus pares y docente a cargo (Cazcarro y Martínez, 2011).

La evaluación consistió en una serie de revisiones, por parte de los/as docentes de las asignaturas respectivas, de las grabaciones de los grupos involucrados en ambas carreras. Una vez que los/as docentes realizaron su evaluación, se recogieron las visiones de cada uno/a para objetivar aún más el criterio final de evaluación. Por otra parte, la realización de los dos procesos mencionados, aplicación del cuestionario de estilos de aprendizaje y la evaluación del esquema discursivo, permitió obtener información

importante del/la estudiante, como el puntaje obtenido en su estilo de aprendizaje, la aprobación en cada una de las etapas y el puntaje total del conjunto de estas en lo que se refiere al esquema discursivo. Al mismo tiempo, se posee información sobre el sexo y carrera de el/la estudiante. La información obtenida del cuestionario y de las observaciones se registró en una planilla digital utilizando el programa SPSS, permitiendo realizar un análisis descriptivo y correlacional de los datos para la presentación de resultados.

\section{ANÁLISIS Y RESULTADOS}

\subsection{ESTILOS DE APRENDIZAJE}

Se puede desprender del resultado descriptivo (Tabla 3) entre los estilos de aprendizaje, que los/as estudiantes de Ingeniería Comercial, en media, poseen mejores puntajes que los/as estudiantes de Pedagogía en Educación Básica, no obstante, no existen diferencias significativas entre estos. 
Tabla 3. Resultado descriptivo de estilos de aprendizaje

\begin{tabular}{|c|c|c|c|c|c|}
\hline $\begin{array}{c}\text { Estilo de } \\
\text { Aprendizaje }\end{array}$ & Carrera & $\begin{array}{l}\text { Número de } \\
\text { estudiantes }\end{array}$ & Media & $\begin{array}{c}\text { Desviación } \\
\text { Estándar }\end{array}$ & $\begin{array}{c}\text { Error } \\
\text { Estándar }\end{array}$ \\
\hline \multirow[t]{3}{*}{ Activo } & $\begin{array}{l}\text { Ingeniería } \\
\text { Comercial }\end{array}$ & 16 & 11,91 & 2,80 & 0,70 \\
\hline & $\begin{array}{c}\text { Pedagogía } \\
\text { Básica }\end{array}$ & 16 & 11,36 & 3,25 & 0,81 \\
\hline & Total & 32 & 11,63 & 3,00 & 0,53 \\
\hline \multirow[t]{3}{*}{ Reflexivo } & $\begin{array}{l}\text { Ingeniería } \\
\text { Comercial }\end{array}$ & 16 & 14,25 & 2,22 & 0,55 \\
\hline & $\begin{array}{c}\text { Pedagogía } \\
\text { Básica }\end{array}$ & 16 & 14,38 & 2,82 & 0,70 \\
\hline & Total & 32 & 14,32 & 2,50 & 0,44 \\
\hline \multirow[t]{3}{*}{ Teórico } & $\begin{array}{l}\text { Ingeniería } \\
\text { Comercial }\end{array}$ & 16 & 14,55 & 2,37 & 0,59 \\
\hline & $\begin{array}{c}\text { Pedagogía } \\
\text { Básica }\end{array}$ & 16 & 12,73 & 4,03 & 1,00 \\
\hline & Total & 32 & 13,64 & 3,38 & 0,60 \\
\hline \multirow[t]{3}{*}{ Pragmático } & $\begin{array}{l}\text { Ingeniería } \\
\text { Comercial }\end{array}$ & 16 & 14,01 & 2,32 & 0,58 \\
\hline & $\begin{array}{c}\text { Pedagogía } \\
\text { Básica }\end{array}$ & 16 & 12,10 & 3,15 & 0,79 \\
\hline & Total & 32 & 13,05 & 2,89 & 0,51 \\
\hline
\end{tabular}

A continuación, en el Gráfico 1, podemos observar que la diagonal principal del cuadrilátero divide a este en dos triángulos cuyas áreas difieren y la mayor corresponde a la del triángulo inferior $(205,5915)$. Esto nos indica que estos estudiantes poseen mejores indicadores hacia los estilos de aprendizaje pragmático, teórico y reflexivo, es decir, son estudiantes que se caracterizan por aplicar lo aprendido, aplicando de manera lógica y coherente teorías, modelos y/o principios, considerando y observando previamente todas las alternativas posibles antes de tomar una decisión. 


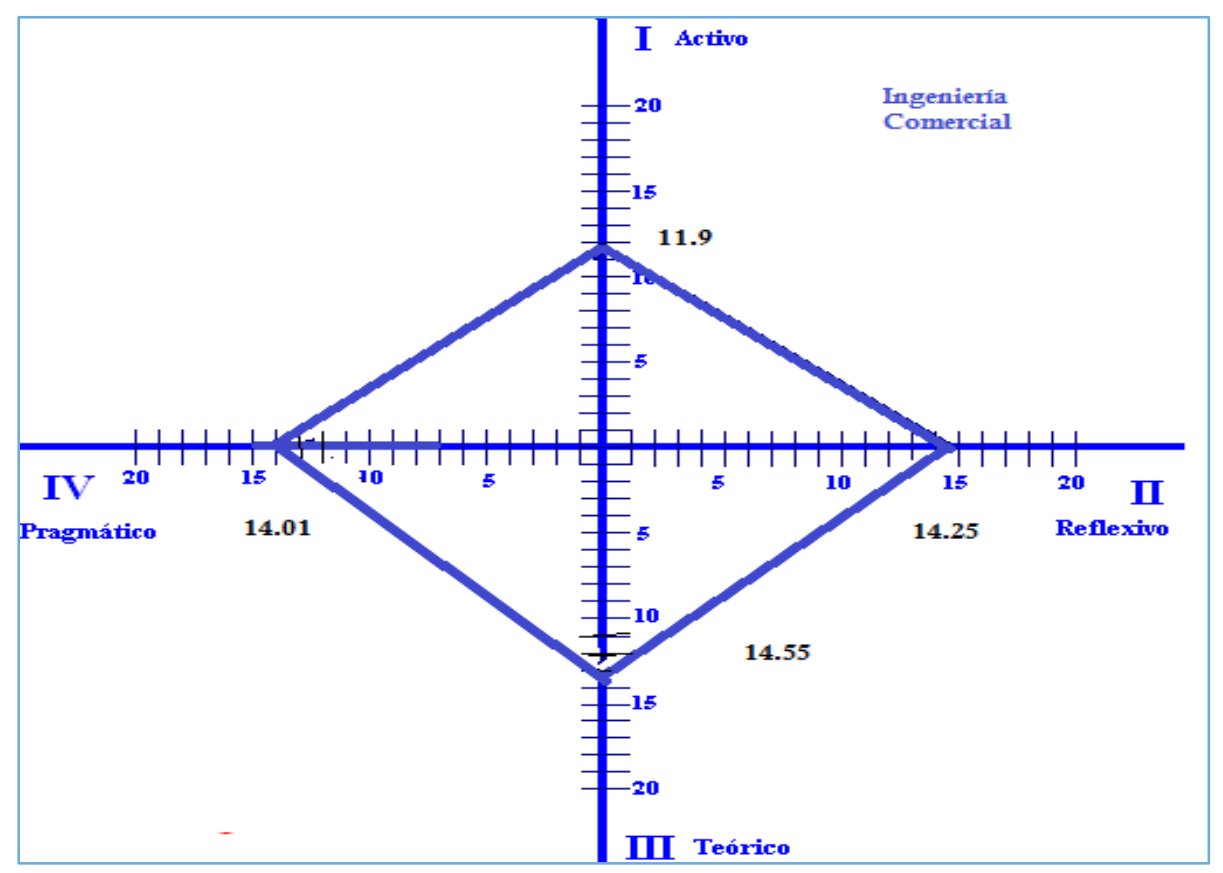

Gráfico 1. Representación de los estilos de aprendizaje de los/as estudiantes de Ingeniería Comercial.

De igual manera, en el Gráfico 2, se puede observar que la diagonal secundaria del cuadrilátero divide a este en dos triángulos, "derecho e izquierdo", en cuyo caso el área del triángulo derecho es mayor $(173,1352)$ que el triángulo izquierdo, indicando que los y las estudiantes de Pedagogía en Educación Básica poseen mejores indicadores en los estilos de aprendizaje activo, reflexivo y teórico, es decir, son estudiantes que se caracterizan por trabajar en grupos, participativos y solucionadores de problemas, con un alto desarrollo de la capacidad de observación y de escucha; además abordan situaciones complejas con fundamentos lógicos. 


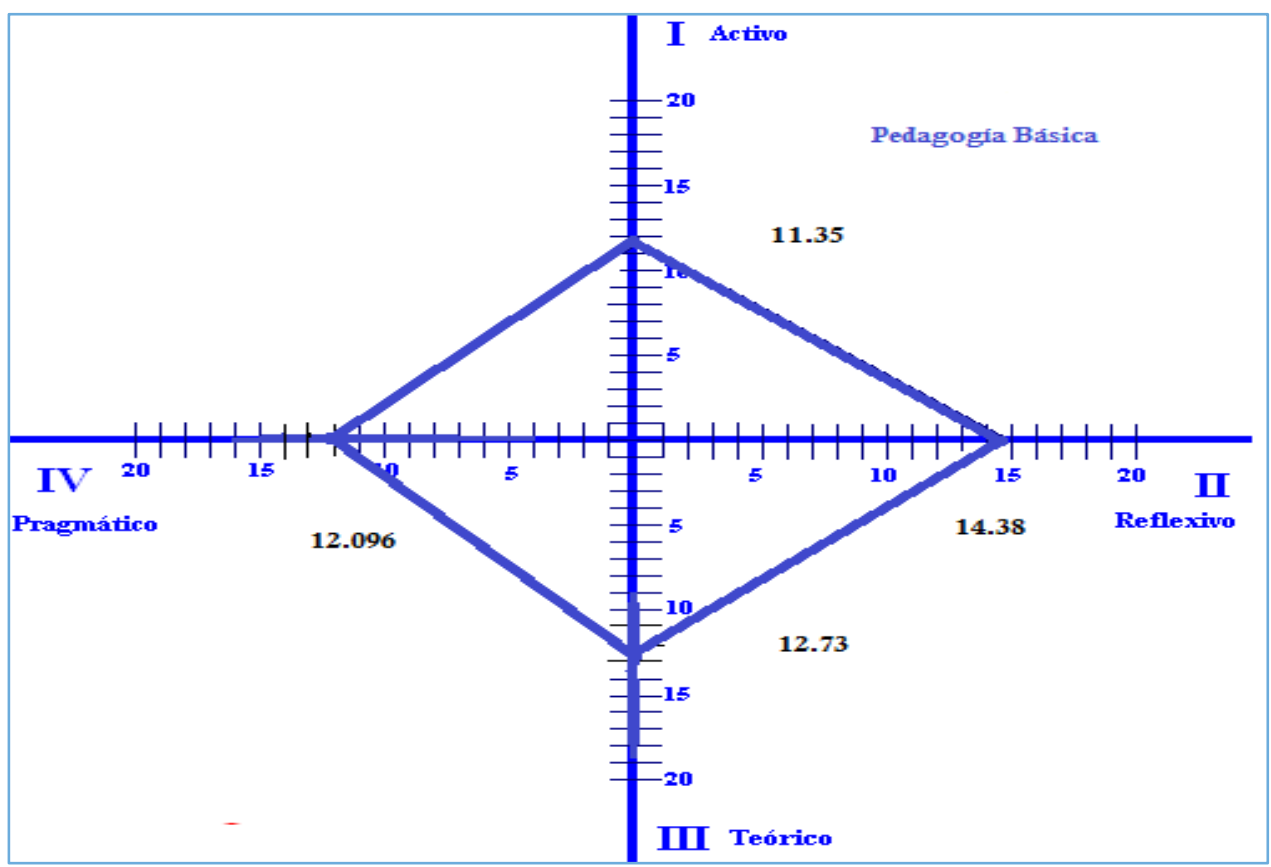

Gráfico 2. Representación de los estilos de aprendizaje de los/as estudiantes de Pedagogía en Educación Básica.

Al comparar ambas muestras se observa que, en cuanto al área obtenida en sus respectivos estilos de aprendizaje, el valor más alto se obtiene en los/as estudiantes de Ingeniería Comercial, cuyo resultado es 205,5915, si se compara con los/as estudiantes de Pedagogía en Educación Básica, cuyo resultado es 173,1352. Esto nos permite evidenciar que los/as estudiantes de Ingeniería tienen un mejor desempeño, en general, de los estilos de aprendizaje. Es decir, los/as estudiantes de Pedagogía en Educación Básica presentan en todos sus estilos un comportamiento moderado de acuerdo a la clasificación de Tabla 1 y los resultados obtenidos en promedio, observados en la Tabla 2, a diferencia de los/as estudiantes de Ingeniería Comercial, quienes presentan niveles moderados a niveles altos, como en los estilos teórico y pragmático.

Esto nos sugiere que en los/as estudiantes de Pedagogía, se debe trabajar en mayor medida actividades que refuercen los estilos de aprendizaje activo, pragmático y teórico. Esto es, ofrecerles actividades donde el rol de lo práctico es claro y destacable, donde se dé un sentido de pertinencia y utilidad a los aprendizajes que se desean alcanzar, todo ello en un marco analítico, reflexivo y crítico. En los/as estudiantes de Ingeniería se sugiere 
reforzar el estilo activo, actividades que proponga desafíos actuales al estudiante, considerando la complejidad de la realidad, lo incierto y lo imprevisto en los contextos de este nuevo siglo.

Por otra parte, al utilizar el criterio de la tabla Baremo (Tabla 2), podemos observar en la Tabla 4, que los/as estudiantes de Ingeniería Comercial evidencian preferencia hacia el estilo de aprendizaje teórico (56\%), lo que nos sugiere que son personas metódicas, lógicas, objetivas, críticas y estructuradas. Este resultado difiere de investigaciones respecto del área de las ciencias económicas (Contreras, 2007), que señalan que el estilo predominante es el activo, no obstante, los estilos que le siguen en menor grado a este grupo son pragmático y activo.

Tabla 4. Porcentaje de estilos de aprendizaje de estudiantes de Ingeniería Comercial.

\begin{tabular}{lc}
\hline Estilo de Aprendizaje & $\begin{array}{l}\text { Porcentaje de } \\
\text { estudiantes(Ing. } \\
\text { Comercial) }\end{array}$ \\
\hline ACTIVO & 13 \\
PRAGMATICO & 31 \\
TEORICO & 56 \\
Total & 100 \\
\hline
\end{tabular}

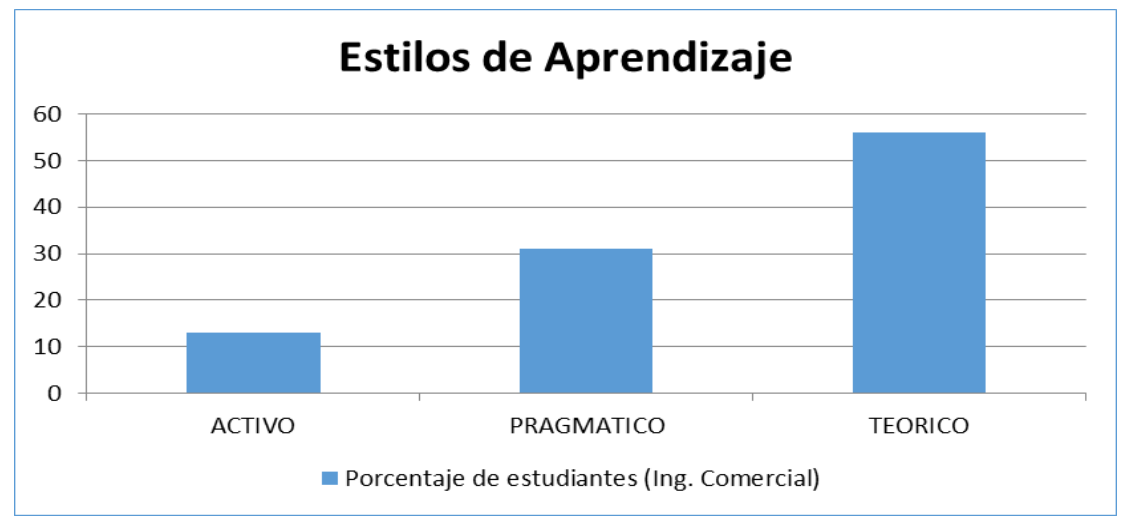

Gráfico 3. Porcentaje de estudiantes de Ingeniería Comercial, según estilo de aprendizaje. 
Ahora bien, al observar los puntajes promedios obtenidos por los/as estudiantes de Ingeniería Comercial y utilizando la distribución de puntajes de la Tabla 2, se obtuvo que los estilos predominantes de estos resultaron ser pragmático, teórico y reflexivo. No obstante, al utilizar la clasificación de acuerdo a la mayor concentración de área (Gráfico 1), se produce un cambio en los estilos de aprendizaje de estos estudiantes a pragmático, teórico y activo. Esto se debe a que los rangos de puntaje en la tabla Baremo no se distribuyen de igual manera por estilo de aprendizaje.

Por otra parte, como se puede observar en la Tabla 5, los/as estudiantes de Pedagogía en Educación Básica tienden hacia el estilo reflexivo, que corresponde a personas que observan y escuchan, y no actúan hasta estar seguros. Estas personas se caracterizan, además, por ser ponderadas, concienzudas, receptivas, analíticas y exhaustivas. Las personas pertenecientes a este estilo de aprender prefieren observar y reflexionar, llevar su propio ritmo de trabajo, tener tiempo para asimilar, oír los puntos de vista de otros/as y realizar análisis detallados. Al mismo tiempo, les resulta dificultoso ocupar el primer plano, actuar de líder, presidir reuniones o debates, participar en reuniones sin planificación, expresar ideas espontáneamente, ser presionadas por el tiempo, verse obligadas a cambiar de una actividad a otra y no tener datos suficientes para sacar conclusiones. Este resultado es coincidente con estudios de estilos de aprendizajes asociados a la educación (Gómez del Valle, 2003). Sin embargo, a estos dos estilos predominantes en este tipo de carrera le siguen en menor grado los estilos activo y teórico.

Tabla 5. Porcentaje de estilos de aprendizaje de estudiantes de Educación en Pedagogía Básica.

\begin{tabular}{lc}
\hline $\begin{array}{l}\text { Estilo de } \\
\text { Aprendizaje }\end{array}$ & $\begin{array}{l}\text { Porcentaje de } \\
\text { estudiantes (Ped. } \\
\text { Básica) }\end{array}$ \\
\hline ACTIVO & 31,25 \\
REFLEXIVO & 56,25 \\
TEORICO & 12,5 \\
Total & 100 \\
\hline
\end{tabular}


Al realizar el análisis comparativo de medias (Test de Kruskal Wallis) entre la carrera y el resultado en el puntaje del estilo de aprendizaje, se obtuvo un p-valor de 0.03 (aproximado), lo que con un nivel de significancia del 10\% permite establecer que existe relación entre el estilo de aprendizaje y la carrera en cuestión. Es decir, se puede establecer que el factor carrera incide en el resultado promedio obtenido en el puntaje de estilo de aprendizaje.

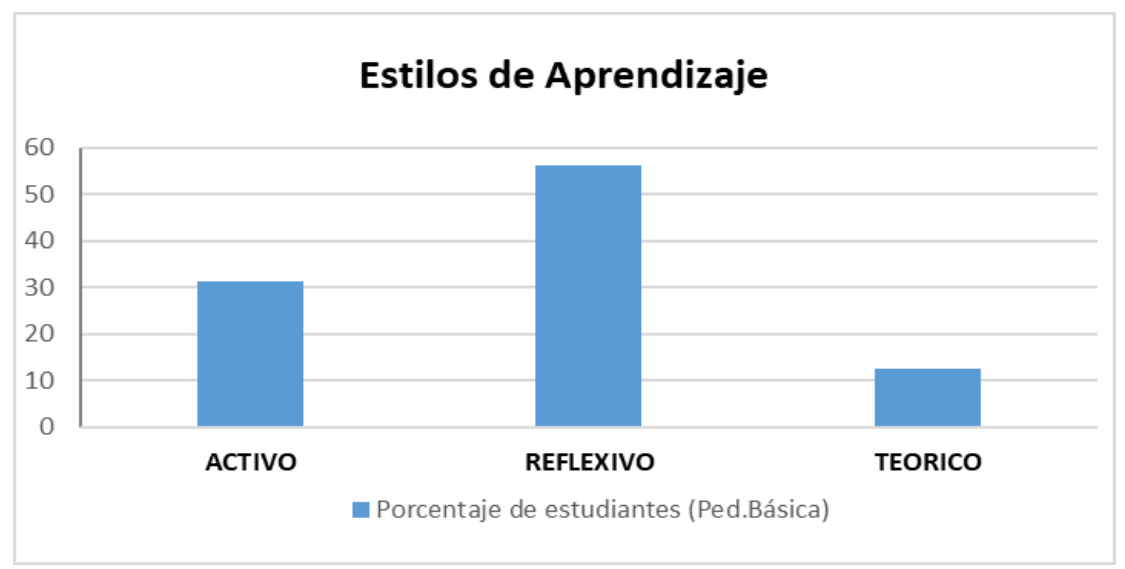

Gráfico 4. Porcentaje de estudiantes de Ped. Básica, según su estilo de aprendizaje.

Finalmente, al realizar comparaciones entre el sexo y el puntaje obtenido en el estilo de aprendizaje (total de alumnos), no existe diferencia significativa en las medias respectivas.

\subsection{SECUENCIA EXPLICATIVA}

En la evaluación de la secuencia explicativa se consideró evaluar con puntaje 0 a aquellos/as estudiantes que omitieron o hicieron mal uso de los marcadores discursivos en la organización de la exposición. A los/as estudiantes que evidenciaron un encadenamiento lógico de las proposiciones, tanto a nivel de estrategia como de marcadores, se consideró otorgar 1 punto. De esta manera, en cada etapa del esquema discursivo, los/as estudiantes contemplan un valor 0 o un valor 1 , dependiendo de los desempeños comunicativos 
expresados. La asignación del puntaje final corresponde a la suma de los resultados obtenidos en cada etapa del esquema discursivo.

A continuación, la Tabla 6 muestra el resultado porcentual del puntaje obtenido por carrera. En ella se observa que los/as estudiantes de Pedagogía en Educación Básica evidencian un logro en la secuencia explicativa concentrándose en puntajes altos, a diferencia de los/as estudiantes de Ingeniería Comercial

Tabla 6. Resultados en la secuencia explicativa entre ambas carreras de acuerdo a la matriz analítica.

\begin{tabular}{ccc}
\hline Puntaje & $\begin{array}{c}\text { Porcentaje de logro en } \\
\text { la secuencia } \\
\text { explicativa. I.C }\end{array}$ & $\begin{array}{c}\text { Porcentaje de logro en } \\
\text { la secuencia } \\
\text { explicativa. P.B }\end{array}$ \\
\hline 0 & 6,3 & 0 \\
1 & 37,5 & 18,8 \\
2 & 25 & 18,8 \\
3 & 18,8 & 56,3 \\
4 & 12,5 & 6,3 \\
\hline
\end{tabular}

El gráfico que se presenta a continuación destaca que un 56,3\% de los/as estudiantes de Educación en Pedagogía Básica alcanzan un puntaje de tres puntos, a diferencia de los/as estudiantes de Ingeniería Comercial, con solo un 18,8\%. Es decir, los/as estudiantes de Pedagogía Básica presentan un mejor desempeño al momento de exponer un tema, mientras que los/as estudiantes de Ingeniería Comercial, en el acto explicativo, presentan un desempeño más bien bajo. 


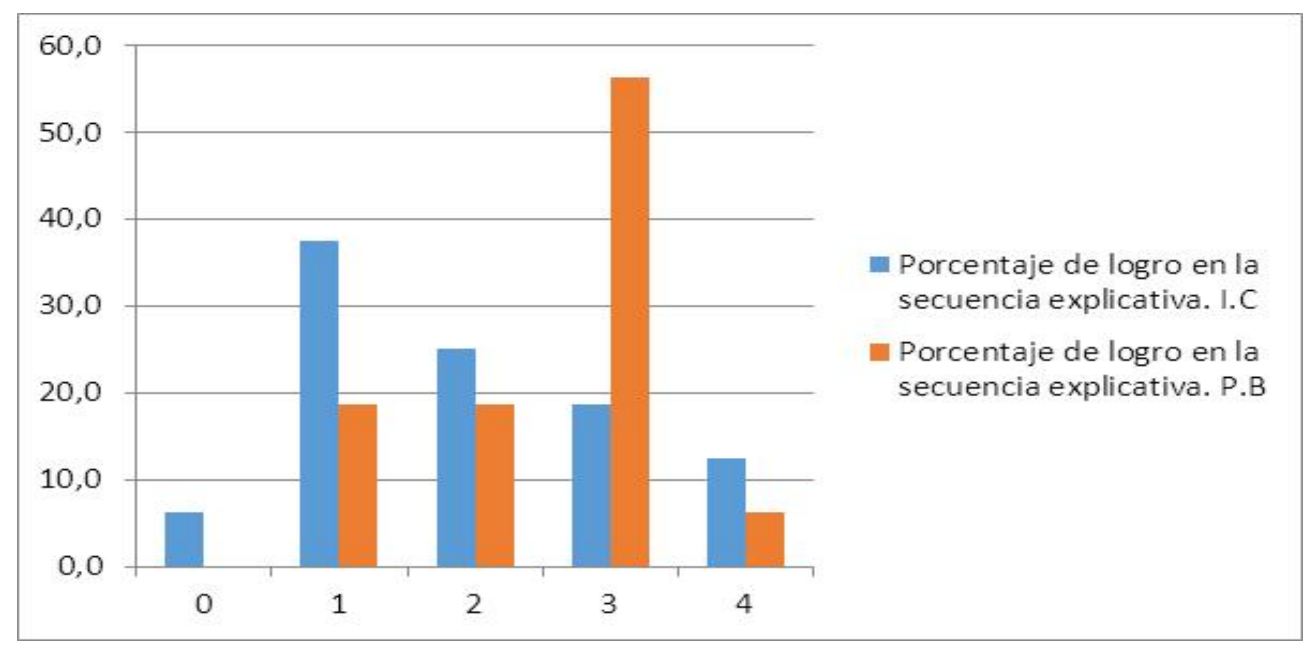

Gráfico 5. Comparación en la secuencia discursiva entre ambas Carreras.

En la siguiente tabla, se presenta el resultado porcentual total de la evaluación obtenida en la secuencia discursiva, observándose diferencias en cada una de las etapas, dependiendo de la carrera.

Tabla 7. Comparación de resultados por etapa en la secuencia discursiva entre ambas carreras.

\begin{tabular}{lcc}
\hline Secuencia Explicativa & Ing. Comercial & Ped. Básica \\
\hline ESQUEMATIZACION & & \\
INICIAL & $56,25 \%$ & $68,75 \%$ \\
PROBLEMA & $31,25 \%$ & $37,50 \%$ \\
EXPLICACIÓN & $75 \%$ & $93,75 \%$ \\
CONCLUSIÓN & $31,25 \%$ & $56,25 \%$ \\
\hline
\end{tabular}

Además, se pudo observar en los videos que los/as estudiantes de Ingeniería Comercial presentan en su actividad discursiva de explicación determinadas estrategias discursivas, como definición, ejemplificación y citación. En cambio, los/as estudiantes de Pedagogía en Educación Básica utilizan variadas estrategias discursivas como definición, reformulación, ejemplificación, analogías y citación. 
En relación a los marcadores discursivos podemos indicar de manera descriptiva que:

1. En la 'esquematización inicial', los/as estudiantes de Pedagogía presentan un desempeño del $68,8 \%$, en relación a un $56,6 \%$ de los/as estudiantes de Ingeniería Comercial. Este resultado se debe a que los marcadores discursivos que contribuyen a dar inicio a la organización del discurso - como por ejemplo, "para empezar", "en primer lugar", "antes que nada", "como es sabido por todos nosotros" - son más recurrentes.

2. En el 'subesquema problema' se evidencia que tanto los/as estudiantes de Ingeniería, con 31,3\% de logro, como los/as de Pedagogía, con un 37,5\%, presentan dificultades para utilizar el operador "por qué" o "cómo" para dar cuenta del tema de estudio que van a exponer.

3. En el 'subesquema explicativo'- esto es, en el uso de, por ejemplo, "porque”, "por eso", "por estas razones" - ambos presentan un buen desempeño, destacando los/as estudiantes de Pedagogía con un 93,8\% y los/as de Ingeniería con un 75\%.

4. En el 'subesquema conclusión', los/as estudiantes de Ingeniería alcanzaron un $31,3 \%$ de logro, a diferencia de los/as de Pedagogía, que alcanzan un 56,3\%. Estos últimos se posicionan en la etapa final que sintetiza y confirma lo que se ha dicho anteriormente, a través de las siguientes expresiones: "a modo de conclusión", “como grupo sostenemos", “en definitiva”, “es posible concluir", "para terminar", entre otras.

Los resultados indican que los/as estudiantes de la carrera de Pedagogía en Educación Básica:

i) Obtuvieron un mejor desempeño discursivo en cuanto a estructura y recursos en la actividad explicativa - expositiva.

ii) Reafirman la temprana transferencia de los modelos y esquemas cognitivo-discursivo a los/as estudiantes noveles del área de la educación por parte de los/as profesores que 
han tenido como referentes a lo largo de su trayectoria educacional (en las etapas de enseñanza básica y media).

En el caso de los/as estudiantes de Ingeniería Comercial, su desempeño discursivo, en cuanto a estructura y recursos en la actividad explicativa, se encuentra en proceso de desarrollo. La transferencia de modelos y esquemas cognitivos discursivos se configura a partir del ingreso hacia su comunidad discursiva disciplinar, lo que comparativamente con el/la estudiante de Pedagogía implica que el desarrollo de su competencia comunicativa es más tardío. Los/as estudiantes aprenden mejor cuando se les plantean actividades de aprendizaje en la resolución de situaciones reales o bien cuando pueden combinar o complementar sus estilos de aprendizaje a través de metodologías participativas.

Para establecer comparación entre ambas carreras de acuerdo al proceso discursivo, se tuvo que categorizar la variable puntaje según el criterio de que si un/a estudiante cumple con más de dos puntos obtenidos, significa que presenta conectores en su discurso. A nivel general se asignó un 1, en caso contrario un 0 , obteniéndose un p-valor de 0.077 (aproximado), lo que, con un nivel de significancia del 10\%, establece que existe relación entre el manejo discursivo y la carrera en cuestión.

No obstante, se realizó el análisis correlacional entre estilos de aprendizaje y los elementos discursivos con resultado no significativo.

\section{CONCLUSIONES}

Actualmente, las competencias genéricas o transversales, como por ejemplo "la comunicación oral", no se ha instalado como una necesidad real en los contextos formativos universitarios, integrándola a las estrategias de aprendizaje de los programas de estudio de las diversas disciplinas. Ello daría a entender que el expresarse en forma clara y efectiva ante una audiencia vendría "por defecto", es decir, el hecho de ingresar a la universidad supondría que los/las estudiantes ya han adquirido las habilidades básicas: hablar, escuchar, leer y escribir de una vez y para siempre. 
Sin embargo, este estudio permitió visibilizar diferencias significativas entre la competencia oral de los/as estudiantes de Pedagogía en Educación Básica y los/as de Ingeniería Comercial. A partir del análisis de la secuencia explicativa, llama la atención el bajo nivel de desempeño en la presentación de la macroproposición explicativa "problema", por parte de los/as estudiantes de ambas carreras, así como el escaso uso de marcadores discursivos para organizar la secuencia explicativa.

Pese a lo anterior, los/as estudiantes de Pedagogía presentan un mayor despliegue de estrategias. Este comportamiento se puede relacionar con la motivación del/a profesor/a de Pedagogía en Educación Básica, en el sentido de que sus esquemas están referidos a prototipos de relación directa en el proceso de escolaridad. Esto es de suma importancia si se considera que el/la formador/a de docentes, en el proceso de formación inicial, transfiere poco a poco modelos y esquemas cognitivos-discursivos favoreciendo el perfil de egreso, ya que se puede afirmar que la elección y empleo de ciertas estrategias discursivas por parte de los hablantes está determinada por las características de estos contextos concretos en que se lleva a cabo la enseñanza-aprendizaje (Prados y Cubero, 2005; Cubero, Cubero, Santamaría, de la Mata, Ignacio y Prados, 2008). Ahora bien, preocupa la situación de los/as estudiantes de Ingeniería Comercial, ya que como señalan algunos académicos del área:

ningún curso o investigación ha tocado en profundidad la sinergia entre la comunicación escrita, oral y visual en Ingeniería; no hay estudios sobre el papel de la reflexión sobre la comunicación como parte de la práctica ingenieril, pocos estudios sobre la comunicación en Ingeniería son lo suficientemente extensos y se sabe poco sobre cómo la comunicación en Ingeniería se relaciona con la preparación de los estudiantes para la práctica profesional. (Valencia 2012, p. 44).

En síntesis, se puede concluir que las competencias comunicativas que se esperan de un/a profesor/a de enseñanza básica al término de su formación profesional, origina alguna ventaja respecto a los/as estudiantes de Ingeniería Comercial. En el caso de estos últimos, el 
manejo de las destrezas comunicativas no está definido en el perfil de los estudiantes que ingresan a esta especialidad.

\section{REFERENCIAS}

Adam, J. (1992). Les textes: types et prototypes. Paris: Nathan.

Alonso, C.; Gallego D.; Honey, P. (1994). Los Estilos de Aprendizaje: Procedimientos de diagnóstico y mejora. Bilbao: Ediciones Mensajero.

Calsamiglia, H. (1994). Usos orales: el estudio del discurso oral. Signos. Teoría y práctica de la educación, (12), 18-28. Recuperado de http://www.quadernsdigitals.net/datos/hemeroteca/r_3/nr_51/a_722/722.html

Camarero Suárez, F., Buey, F., y Herrero Díez, J. (2000). Estilos y estrategias de aprendizaje en estudiantes universitarios. Psicothema, (12), 615-622. Recuperado de http://www.redalyc.org/articulo.oa?id=72712416

Carlino, P. (2005). Escribir, Leer y Aprender en la Universidad. Una Introducción a la Alfabetización Académica. Buenos Aires: Fondo de Cultura Económica.

Carlino, P. (2013). Alfabetización académica diez años después. Revista Mexicana de Investigación Educativa, (18), 355-381. Recuperado de http://www.redalyc.org/pdf/140/14025774003.pdf

Casco, M. R. (abril de 2000). Competencias discursivas e integración del estudiante al ámbito universitario. Ponencia llevada a cabo en el $V$ Congreso de ALAIC, Santiago de Chile 
Cazcarro, I. y Martínez-Caraballo, N. (2011). La grabación en vídeo en el aula como herramienta de mejora de la competencia de comunicación oral. Education Siglo $X X I,(29), 255-282$

Contreras, Y. (noviembre de 2007). Los estilos de aprendizaje en los alumnos de nivel superior. Ponencia llevada a cabo IX Congreso Nacional de Investigación Educativa, Yucatán, México

Cubero, R., Cubero, M., Santamaría, A., de la Mata, M., Ignacio, M. y Prados, M. (2008). La educación a través de su discurso. Prácticas educativas y construcción discursiva del conocimiento en el aula. Revista de Educación, (346), 71-104. Recuperado de http://www.revistaeducacion.mec.es/re346/re346_03.pdf

Gallardo Paúls, B. (2007). Pragmática para logopedas. Cádiz, Universidad de Cádiz.

Gómez del Valle, M. (2003). Identificación de los estilos de aprendizaje predominantes en estudiantes de magisterio de la Facultad de Ciencias de la Educación de la Universidad de Cádiz. Revista Electrónica Interuniversitaria de Formación del Profesorado, (6), 1-4. $\quad$ Recuperado de https://dialnet.unirioja.es/servlet/articulo?codigo $=754490$

González, M. (2011). Estilos de aprendizaje: su influencia para aprender a aprender. Revista Estilos de Aprendizaje, (7), 207-216. Recuperado de https://www2.uned.es/revistaestilosdeaprendizaje/numero_7/sumario_completo/lsr_ 7_abril_2011.pdf

Gutiérrez, M., García, J., Vivas. M., Santizo, J., Alonso, C. y Arranz, M. (2011). Estudio Comparativo de los estilos de aprendizajes del alumnado que inicia sus estudios universitarios en diversas Facultades de Venezuela, México y España. Revista de Estilos de Aprendizaje, (7), 35-62. Recuperado de 
https://www2.uned.es/revistaestilosdeaprendizaje/numero_7/sumario_completo/lsr_ 7_abril_2011.pdf

Hymes, D. H. (Ed.). (1964) Language in culture and society: A reader in linguistics and anthropology. New York, Estados Unidos: Harper \& Row.

Hymes, D.H. (1972). On Communicative Competence. En J.B. Pride and J. Holmes (Ed.) Sociolinguistics (pp.269-293). Harmondsworth: Penguin.

Latorre, M., Aravena P., Milos, P. y García, M. (2010). Competencias habilitantes: un aporte para el reforzamiento de las trayectorias formativas universitarias. Revista Calidad en la educación, (33), 275-301. Recuperado de https://dialnet.unirioja.es/servlet/articulo?codigo $=3732194$

López, M. A. (2013). Aprendizaje, competencias y TIC. Aprendizaje basado en competencias. México: Pearson.

Manzano Díaz, C. e Hidalgo Diez, E. (2009). Estilos de aprendizaje, estrategias de lectura y su relación con el rendimiento académico de la lengua extranjera. Revista $\begin{array}{lllll}\text { Educación } & X X 1 & \text { (12), } & \text { 123-150. } & \text { Recuperado de }\end{array}$ http://www.redalyc.org/pdf/706/70611919007.pdf

Martín, E, y Moreno, A. (2007). Competencia para aprender a aprender. Alianza editorial. Madrid.

Prados, M. y Cubero, R. (2005). Construcción del conocimiento y discurso educativo. Una aproximación al estudio del discurso de profesores y alumnos en la universidad Avances en Psicología Latinoamericana, (23), 141-153. Recuperado de http://www.redalyc.org/pdf/799/79902311.pdf 
Tomlin, R., Forrest, L., Pu, M. y Kim, M. (1997). Semántica del discurso. En T. A. van Dijk (Ed.). El discurso como estructura y proceso, (pp. 107 - 170). Barcelona: Editorial Gedisa.

Valencia, A. (2012). El problema de la comunicación en ingeniería: el caso de las universidades, Revista Ingeniería y sociedad, (5), 39-45. Recuperado de https://aprendeenlinea.udea.edu.co/revistas/index.php/ingeso/article/view/13986

Van Dijk, T., y Kintsch, W. (1983). Strategies of Discourse Comprehension. New York: Academic Press.

Vilà I Santasusana, M. (Coord.). (2005). El discurso oral formal. Contenidos de aprendizaje y secuencias didácticas. Barcelona: Editorial Graó.

Villa Sánchez A. y Poblete Ruiz, M. (Ed.). (2007). Aprendizaje basado en competencias. Una propuesta para la evaluación de las competencias genéricas. Recuperado de https://docplayer.es/13727-Aprendizaje-competencias.html

Zamudio, B. y Atorresi, A. (2000). La explicación. Buenos Aires: Eudeba. 